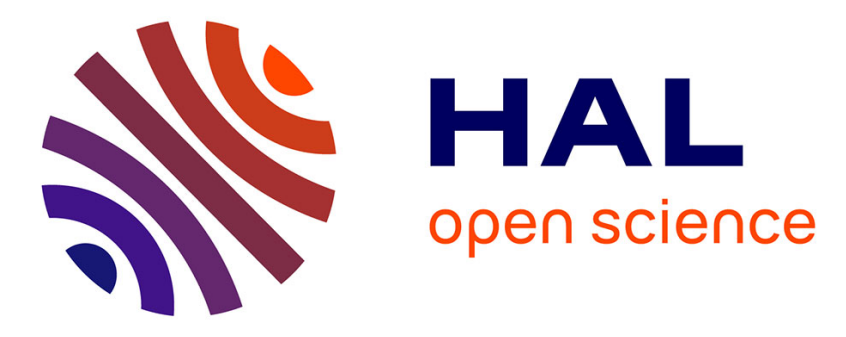

\title{
Set-point reconfiguration approach for the FTC of wind turbines
}

Boumedyen Boussaid, Christophe Aubrun, Naceur Abdelkrim

\section{To cite this version:}

Boumedyen Boussaid, Christophe Aubrun, Naceur Abdelkrim. Set-point reconfiguration approach for the FTC of wind turbines. 18th IFAC World Congress, IFAC WC'2011, Aug 2011, Milan, Italy. pp.12395-12400. hal-00638190

\section{HAL Id: hal-00638190 https://hal.science/hal-00638190}

Submitted on 4 Nov 2011

HAL is a multi-disciplinary open access archive for the deposit and dissemination of scientific research documents, whether they are published or not. The documents may come from teaching and research institutions in France or abroad, or from public or private research centers.
L'archive ouverte pluridisciplinaire HAL, est destinée au dépôt et à la diffusion de documents scientifiques de niveau recherche, publiés ou non, émanant des établissements d'enseignement et de recherche français ou étrangers, des laboratoires publics ou privés. 


\title{
Set-point reconfiguration approach for the FTC of wind turbines
}

\author{
B. Boussaid * C. Aubrun* N. Abdelkrim** \\ * Centre de Recherche en Automatique de Nancy (CRAN), \\ University of Nancy, CNRS, BP 239, 54506 Vandoeuvre Cedex, France \\ (e-mail: \{boussaid.boumedyen,christophe.aubrun\}@cran.uhp-nancy.fr) \\ ** Modelisation, Analyse et Commande des Systemes (MACS), University of \\ Gabes, Omar Ibn Khattab Road, 6029 Gabes, Tunisia (e-mail: \\ naceur.abdelkrim@enig.rnu.tn).
}

\begin{abstract}
In this paper, a two-level reconfiguration action is used to design an FTC system. The objective is to adapt the corrective action accordingly to the fault severity. The first level of the procedure is triggered when the impact of the fault on the system performance is limited. At the second level a dynamic reference modification based on reference-offset governor is proposed. The idea consists of updating the controller by the new post-fault system model in first time and; in second time; modifying the set-point or reference according to the system constraints which become more strict after fault occurrence in order to avoid any actuator saturation and ensure system stability. The effectiveness of the proposed solution is illustrated by a wind turbine example subjects to actuator faults and constrained on the actuator dynamic ranges.
\end{abstract}

Keywords: FTCS; Reference Governor; Actuator fault; Performance degradation.

\section{INTRODUCTION}

In industrial processes, systems to be controlled are becoming more and more complex. One of these complexities relies in the necessity of satisfying input/state constraints which are dictated by physical limitations of the actuators and by the necessity to keep some plant variables within safe limits. In general, these constraints are integrated in the selection of the process equipment and the design of the nominal controllers. Another complexity is the occurrence of faults in plants which make the design of a controller tolerant to faults a very important issue.

Nowadays, wind turbines which generate electrical energy from the wind energy are considered one of these complex systems. The installed turbines are often in megawatt size and are very expensive which need more availability (See Odgaard et al. [2009]). Besides, physical and functional limitations should be kept in safe limits which need to handle with care any abnormal operating especially after fault occurrence. So that, degraded mode is needed to ensure safe functioning.

From Fault-Tolerant Control (FTC) design point of view, the post-fault system should recover the original performance which is usually a quiet ambitious objective. In refs. Jiang and Zhang [2006] and Zhang and Jiang [2003], it is considered that the system can always operate under degraded performance. In practice, once a fault occurs, the degree of the system redundancy and the available actuator capabilities can be significantly reduced. Further, the FTC may lead to actuator saturation, or worse still, to cause damage to the system, and even result in lose of the system stability (See Jiang and Zhang [2002]).

A significant amount of work has been done to deal with actuator saturation. Model predictive control is an effective control algorithm for dealing with actuator saturation (See Mayne et al.
[2000]). Anti-windup research was largely discussed and many constructive design algorithms were formally proved to induce suitable stability properties (See Kothare and Morari [1999],Wu and $\mathrm{Lu}$ [2004]). Other class of framework consists of modifying the reference input so that the constraints are not violated (See Angeli et al. [2001],Bemporad et al. [1997], Casavola et al. [2000],Gilbert et al. [1995],Gilbert and Tan [1991]). These approaches are able to handle input and/or state-related constraints, using methods based on model predictive control ideas, to synthesize Command or Reference Governor (RG). In ref. citekol06, Parameter Governor (PG) unit is proposed which enforces pointwise-in-time constraints on the evolutions of relevant system variables. Later, both Reference Governor (RG) and Parameter Governor (PG) actions are integrated in a single unit as Reference-Offset Governor (ROG) (See Casavola et al. [2007]), which adds many advantages especially in enlarging the set of feasible evolutions of the system. The function of ROG device is to modify, whenever necessary, the reference and add an offset to the nominal control action in order to enforce pointwise-in-time constraints and to improve the overall system transient performance (See Casavola et al. [2006], Casavola et al. [2007]).

In this paper, a new reconfiguration system approach based on Reference-Offset Governor and separate LQ controller is proposed. The reconfiguration capability of ROG unit is basically the respect of system constraint obligations and the dynamic modification of the reference according to an acceptable performance degradation. Furthermore, the stability of the closedloop system is ensured.

The main contribution of this paper is to deal with performance degradation in the case of wind turbines subject to actuator faults. A nonlinear reference management is used to ensure actuators saturation avoidance and system stability with accept- 
able performance degradation via dynamic reference modification.

The paper is organized as follows. The formulation of the problem statement is presented in section 2. Section 3 is reserved to deal with the design of the FTC system. Section 4 is dedicated to illustrate the idea with an example of a wind turbine benchmark followed by simulation results interpretations. Finally, the paper is ended by a conclusion.

\section{PROBLEM FORMULATION}

\subsection{Standard LQ Control}

Let us consider the following Linear Time-Invariant (LTI) system in discrete time

$$
\left\{\begin{aligned}
x(t+1) & =A x(t)+B u(t)+G_{d} d(t) \\
y(t) & =C x(t)
\end{aligned}\right.
$$

Where $x(k) \in \mathscr{R}^{n}$ is the state vector, $u(k) \in \mathscr{R}^{m}$ is the input vector, $y(k) \in \mathscr{R}^{p}$ is the output vector, $d(t) \in \mathscr{R}^{n_{d}}$ is an exogenous bounded disturbance and $\left(A, B, C, G_{d}\right)$ represents the system dynamics.

Let rank $(C)=p$ and $\operatorname{rank}(B)=m \geq p$. Assume that the full-state $x$ is available. By solving the Linear Quadratic Regulation problem (See Staroswiecki [2003], Harkegard and Glad [2005]), the optimal control law is given by :

$$
u(t)=-K x(t)+K_{r} r(t)
$$

with

$$
\begin{aligned}
K & =R^{-1} B^{T} P \\
K_{r} & =R^{-\frac{1}{2}}\left(C(B K-A)^{-1} B R^{-\frac{1}{2}}\right)^{+}
\end{aligned}
$$

where $Q$ is a positive semi-definite matrix and $R$ is a positive definite matrix. $Q$ and $R$ are preselected by the designer to achieve the nominal performance. $P$ is a unique positive semidefinite and symmetric solution of the Algebraic Riccati Equation (ARE)

$$
A^{T} P+P A+Q-P B R^{-1} B^{T} P=0
$$

\subsection{Limitation of the LQ solution in constrained control}

Assume that one has constraints on control signal, so one should use a block to limit input signal to the upper and lower saturation values (see Fig. 1).

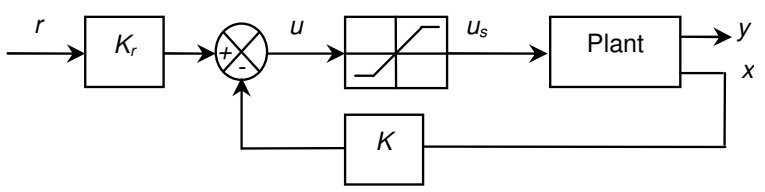

Fig. 1. LQ Regulator with input signal limitation block

The new control signal with the consideration of a piecewiselinear saturation is described as

$$
u_{s}=\sigma(u)= \begin{cases}u_{j, \max } & \text { if } u_{j} \geq u_{j, \max } \\ u_{j} & \text { if } u_{j, \min } \leq u_{j} \leq u_{j, \max } \\ u_{j, \min } & \text { if } u_{j} \leq u_{j, \min }\end{cases}
$$

for $j=1,2, \ldots, m$ and $m$ is the number of actuators.
Ideally, the nominal LQ Controller is designed to work inside the allowed control limit. However, a slight saturation is possible during short time without destabilizing the system. In faulty case, these constraints become more strict and the probability to reach the saturation region increases with the fault severity especially in the case of loss of actuator effectiveness which may destabilize the system or induce severe performance degradation through a decrease of feedback gain, Kapasouris et al. [1988].

In general, nonlinear control laws are required to stabilize linear systems subject to input saturation and, for more generality, subject to input/state constraints. Our approach consists in associating a reconfigurable Reference Offset-Governor block to a reconfigurable LQ Regulator in order to solve the problem of actuator saturations and input/state constraints in general. Besides, the performance degradation is taken account by modifying the references by the ROG unit with the consideration of system constraints and nominal objectives.

\subsection{Reference-Offset Governor solution}

Let us consider the global system including the ROG unit and the feedback controller, as depicted in Fig. 2.

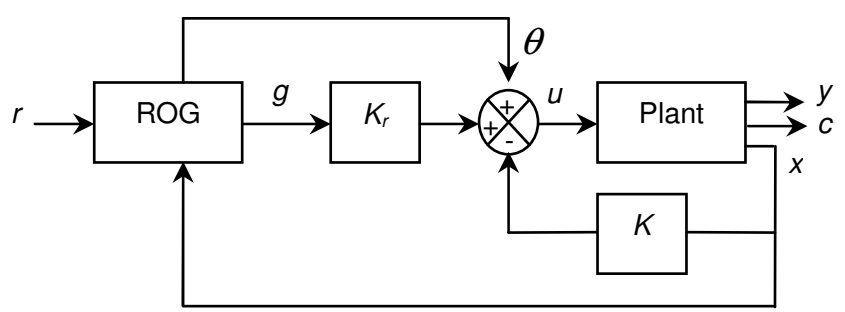

Fig. 2. Global system diagram including Controller and ROG blocks

According to Fig. 2, the control input can be written as :

$$
\begin{aligned}
u(t) & =-K x(t)+K_{r} g(t)+\theta(t) \\
& =-K x(t)+K_{z} z(t)
\end{aligned}
$$

where $K_{z}=\left[\begin{array}{ll}K_{r} & I_{m}\end{array}\right]$ and $z(t)=\left[\begin{array}{ll}g(t) & \theta(t)\end{array}\right]^{T}$. One replaces (7) in (1), one gets

$$
\begin{aligned}
x(t+1) & =(A-B K) x(t)+B K_{z} z(t)+G_{d} d(t) \\
& =\Phi x(t)+G z(t)+G_{d} d(t)
\end{aligned}
$$

Where $\Phi=(A-B K)$ and $G=B K_{z}$.

Besides, if one considers only the control input constraints, and one puts $H_{c}=-K$ and $L=K_{z}$

$$
c(t)=H_{c} x(t)+L z(t)+L_{d} d(t)
$$

So, the LTI system in (1) becomes

$$
\left\{\begin{aligned}
x(t+1) & =\Phi x(t)+G z(t)+G_{d} d(t) \\
y(t) & =H_{y} x(t) \\
c(t) & =H_{c} x(t)+L z(t)+L_{d} d(t)
\end{aligned}\right.
$$

with $x(t) \in \mathscr{R}^{n}$ the state vector which includes the controller states; $g(t) \in \mathscr{R}^{p}$ the manipulable reference which would essentially coincide with the reference $r(t) \in \mathscr{R}^{p} ; \theta(t) \in \mathscr{R}^{m}$ an adjustable offset on the nominal control law which is assumed to be selected from a given convex and compact set $\Theta$, 
with $0_{m} \in \operatorname{int} \Theta ; d(t) \in \mathscr{R}^{n_{d}}$ an exogenous bounded disturbance satisfying $d(t) \in \mathscr{D} ; \forall t \in \mathscr{Z}_{+}$with $\mathscr{D}$ a specified convex and compact set such that $0_{n_{d}} \in \mathscr{D} ; y(t) \in \mathscr{R}^{p}$ the output, viz. a performance related signal; $c(t) \in \mathscr{R}^{n_{c}}$ the constraints vector, $c(t) \in \mathscr{C} ; \forall t \in \mathscr{Z}_{+}$; with $\mathscr{C} \subset \mathscr{R}^{n_{c}}$ a prescribed constrained set. It is assumed that:

A.1) $\Phi$ is a stable matrix;

A.2) System (10) is offset-free w.r.t. $g(t)$ i.e.

$$
H_{y}\left(I_{n}-\Phi\right)^{-1} G_{g}=I_{p}
$$

where $z(t)=\left[\begin{array}{ll}g(t) & \theta(t)\end{array}\right]^{T} \in \mathscr{R}^{p+m}$, is the ROG output and the following matrices are defined $G=\left[\begin{array}{ll}G_{g} & G_{\theta}\end{array}\right], L=\left[\begin{array}{ll}L_{g} & L_{\theta}\end{array}\right]$.

The ROG design problem consists of generating, at each time $t$; the command input $z(t)$ as an algebraic function of the current state $x(t)$ and reference $r(t)$

$$
z(t):=\bar{z}(x(t), r(t))
$$

The ROG output is based on the minimization of a cost function subject to prescribed constraints. The cost function has the following form

$$
J(x(t), z(t), r)=\|g(t)-r\|_{\Psi_{g}}^{2}+\|\theta(t)\|_{\Psi_{\theta}}^{2}
$$

where $\Psi_{g}=\Psi_{g}^{T}>0_{m}, \Psi_{\theta}=\Psi_{\theta}^{T}>0_{m}$ and $\|v\|_{\Psi}^{2}:=v^{T} \Psi v$. Thus, at each time $t \in \mathscr{Z}_{+}$, the ROG output is chosen according to the solution of the following constrained optimization problem, Casavola et al. [2007],

$$
z(t):=\arg \min _{z \in \mathscr{V}(x(t))} J(x(t), z(t), r)
$$

\section{FTC SYSTEM DESIGN}

Let us consider the following proposed scheme in FTC solution (See Fig. 3).

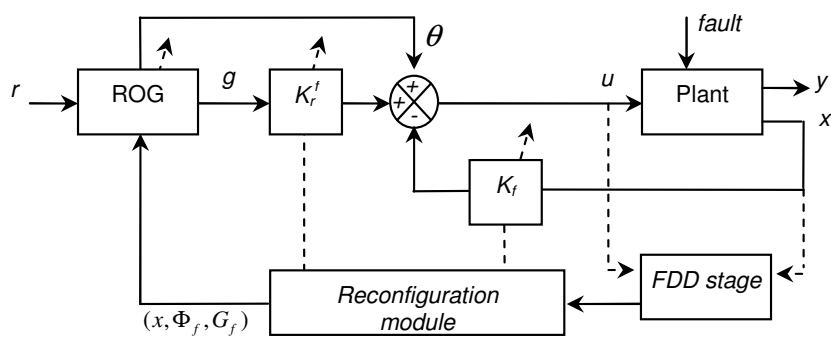

Fig. 3. Proposed scheme of FTC system design

The bloc FDD is assumed perfect and the matrices $A_{f}$ and $B_{f}$ of the system post-fault are detectable.

\subsection{Controller reconfiguration design}

After the fault occurrence and the FDD action, the updated space state representation of the system is

$$
\left\{\begin{aligned}
x(t+1) & =A_{f} x(t)+B_{f} u(t) \\
y(t) & =C x(t)
\end{aligned}\right.
$$

Assuming that the system post-fault is controllable and $\left(A_{f}, B_{f}\right)$ is still stabilizable, from the Bellman's optimality principle, the optimal reconfigurable strategy (See Staroswiecki [2003]) consists in applying a new optimal control action to the system (14)

$$
u_{f}(t)=-K_{f} x(t)+K_{r}^{f} r(t)
$$

with

$$
\begin{aligned}
K_{f} & =R^{-1} B_{f}^{T} P_{f} \\
K_{r}^{f} & =R^{-\frac{1}{2}}\left(C\left(B_{f} K_{f}-A_{f}\right)^{-1} B_{f} R^{-\frac{1}{2}}\right)^{+}
\end{aligned}
$$

where $P_{f}$ is a unique positive semi-definite and symmetric solution of the Algebraic Riccati Equation (ARE)

$$
A_{f}^{T} P_{f}+P_{f} A_{f}+Q-P_{f} B_{f} R^{-1} B_{f}^{T} P_{f}=0
$$

\subsection{ROG reconfiguration design}

Hereafter, an extension of the ROG principle in the faulty case is proposed. It is assumed that the system post-fault dynamic is $\left(A_{f}, B_{f}\right)$ and the controller feedback and feed-forward gains are denoted $K_{f}$ and $K_{r}^{f}$.

In closed-loop scheme, the system state space representation is given by

$$
\begin{aligned}
x(t+1) & =A_{f} x(t)+B_{f} u(t)+G_{d} d(t) \\
& =\left(A_{f}-B_{f} K_{f}\right) x(t)+B_{f}\left(K_{r}^{f} g(t)+I_{m} \theta(t)\right)+G_{d} d(t)
\end{aligned}
$$

Thus, the state space description of the plant (19) becomes :

$$
\left\{\begin{aligned}
x(t+1) & =\Phi_{f} x(t)+G_{f} z(t)+G_{d} d(t) \\
y(t) & =H_{y} x(t) \\
c(t) & =H_{c}^{f} x(t)+L_{f} z(t)+L_{d} d(t)
\end{aligned}\right.
$$

where

$$
\begin{aligned}
& \Phi_{f}=A_{f}-B_{f} K_{f} \\
& G_{f}=B_{f} K_{z}^{f} \\
& K_{z}^{f}=\left[\begin{array}{ll}
K_{r}^{f} & I_{m}
\end{array}\right]
\end{aligned}
$$

with $\Phi_{f}$ and $G_{f}$ represented the global system dynamics in closed-loop after fault occurrence.

Considering the ROG unit in the faulty case as shown in Fig. 3, and we assume that :

B.1) $\Phi_{f}$ is a stable matrix;

B.2) system (20) is offset-free w.r.t. $g(t)$ i.e.

$$
H_{y}\left(I_{n}-\Phi_{f}\right)^{-1} G_{g}^{f}=I_{p}
$$

The solution of the cost function (12) is :

$$
z(t):=\arg \min _{z \in \mathscr{V}_{f}(x(t))} J(x(t), z(t), r)
$$

with $\mathscr{V}_{f}(x(t))$ is the set of the disturbance-free virtual evolution of the constraints vector $\bar{c}_{f}(k, x(t), z)$ after fault occurrence

$$
\mathscr{V}_{f}(x(t))=\left\{z \in \mathscr{W}_{\delta}^{f}: \bar{c}_{f}(k, x(t), z) \in \mathscr{C}_{k}^{f}, \forall k \in \mathscr{Z}_{+}\right\}
$$

where $\bar{c}_{f}(k, x(t), z)$ is given by :

$$
\bar{c}_{f}(k, x(t), z)=H_{c}^{f}\left(\Phi_{f}^{k} x(t)+\sum_{i=0}^{k-1} \Phi_{f}^{k-i-1} G_{f} z\right)+L_{f} z
$$

and the two sets; $\mathscr{W}_{\delta}^{f}$ and $\mathscr{C}_{f}^{\delta}$; are given by : 


$$
\begin{aligned}
\mathscr{W}_{\delta}^{f} & :=\left\{z \in \mathscr{R}^{p+m}: \bar{c}_{z} \in \mathscr{C}_{f}^{\delta}\right\} \\
\mathscr{C}_{f}^{\delta} & :=\mathscr{C}_{\infty}^{f} \sim \mathscr{B}_{\delta}
\end{aligned}
$$

Note that $\mathscr{C}_{\infty}^{f}$ is constructed from recursive sets $\mathscr{C}_{k}^{f}$

$$
\mathscr{C}_{\infty}^{f}:=\bigcap_{k=0}^{k_{0}^{f}} \mathscr{C}_{k}^{f}
$$

where the sets $\mathscr{C}_{k}^{f}$ are defined from $k \in\left\{0,1, \ldots, k_{0}^{f}\right\}$ as

$$
\begin{aligned}
\mathscr{C}_{0}^{f} & :=\mathscr{C}^{f} \sim L_{d} \mathscr{D} \\
& \vdots \\
\mathscr{C}_{k}^{f} & :=\mathscr{C}_{k-1}^{f} \sim H_{c}^{f} \Phi_{f}^{k-1} G_{d} \mathscr{D}
\end{aligned}
$$

with $\mathscr{C}^{f}$ is the prescribed constrained set after fault occurrence. The following properties hold true for the above described ROG in faulty case.

Theorem 1. Let assumptions (B.1) be fulfilled. Consider system (20) along with the ROG selection rule (24), and let $\mathscr{V}_{f}(x(0))$ be non-empty. Then:

1. The minimizer in (24) uniquely exists at each $t \in \mathscr{Z}_{+}$and can be obtained by solving a convex constrained optimization problem, viz. $\mathscr{V}_{f}(x(0))=\mathscr{V}\left(x\left(t_{f}\right)\right)$ non-empty implies $\mathscr{V}_{f}(x(t))$ non-empty along the trajectories generated by the ROG command (20). Such the time of fault occurrence $t_{f}$ is determined by the FDD stage.

2. The set $\mathscr{V}_{f}(x(t)), \forall x(t) \in \mathscr{R}^{n}$, is finitely determined, viz. there exists an integer $k_{0}^{f}$ such that if $\bar{c}_{f}(k, x(t), z) \in \mathscr{C}_{k}^{f}, k \in$ $\left\{0,1, \ldots, k_{0}^{f}\right\}$, then $\bar{c}_{f}(k, x(t), z) \in \mathscr{C}_{k}^{f} \forall k \in \mathscr{Z}_{+}$. Such a constraint horizon $k_{0}^{f}$ can be determined off-line.

3. The constraints are fulfilled for all $t \in \mathscr{Z}_{+}$.

4. The overall system is asymptotically stable; in particular, whenever $r(t) \equiv r, \lim _{t \rightarrow \infty} \theta(t)=0_{m}$, and $g(t)$ converges either to $r$ or to its best steady-state admissible approximation $\hat{r}$, with

$$
\hat{z}(t):=\left[\begin{array}{ll}
\hat{r} & 0_{m}
\end{array}\right]^{T}:=\arg \min _{z \in \mathscr{V}(x(t))} J(x(t), z(t), r)
$$

Consequently, by the offset-free condition (B.2), $\lim _{t \rightarrow \infty} \bar{y}(t)=\hat{r}$, where $\bar{y}$ is the disturbance-free component of $y$.

Proof. The proof is similar to that presented in Casavola et al. [2007].

\section{WIND TURBINES}

\subsection{Wind turbine description and model}

In this paper, a specific variable speed turbine is considered. It is a three blade horizontal axis turbine with a full converter. The energy conversion from wind energy to mechanical energy can be controlled by changing the aerodynamics of the turbine by pitching the blades or by controlling the rotational speed of the turbine relative to the wind speed. The mechanical energy is converted to electrical energy by a generator fully coupled to a converter. Between the rotor and the generator a drive train is used to increase the rotational speed from the rotor to the generator. The objective of the control system is to track the power reference while keeping the mechanical vibrations to minimal value. A more detailed description of the general function of the wind turbine can be seen in Odgaard et al. [2009].

In principle, wind turbine system can be divided in three sub-systems: blade and pitch system, drive train system and Generator/conversion system. Fig. 4 shows the relation-ships between each sub-system and the controller, see e.g Odgaard et al. [2009]. The turbine controller operates in four zones. Zone 1 is start up of the turbine, Zone 2 is power optimization, Zone 3 is constant power production and Zone 4 is high wind speed, see e.g. Johnson et al. [2006]. The focus of this work is on the normal operation consequently only Zone 3 is considered. In this zone the control objective is to follow the power reference.

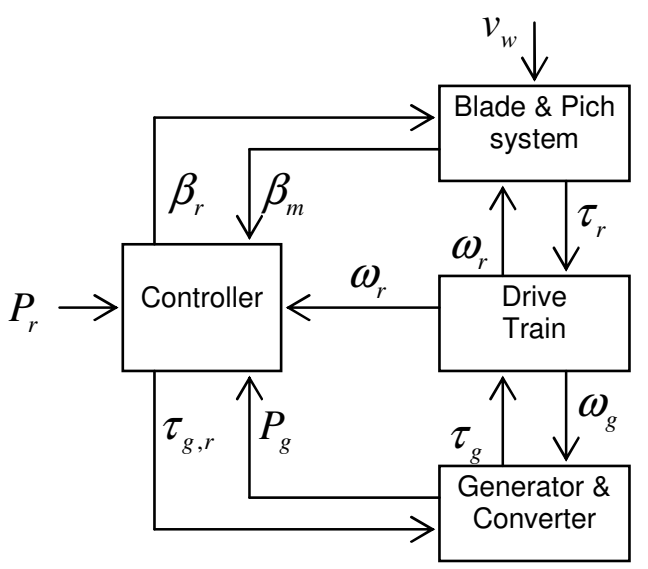

Fig. 4. Simplified wind turbine diagram.

The aerodynamics of the wind turbine is modeled as torque acting on the blades. This aerodynamic torque, $\tau_{r}$, can be represented by, see Odgaard et al. [2009]:

$$
\tau_{r}(t)=\sum_{j=1}^{j=3} \frac{\rho \pi R^{3} C_{q}\left(\lambda(t), \beta_{j}(t)\right) v_{w}(t)^{2}}{6}
$$

where $C_{q}$ is the torque coefficient, $\lambda$, is the tip speed ratio, $\rho$, is the air density, $R$, is the radius of blades, $v_{w}$, is the wind speed and $\beta_{j}$, is the pitch angle referring to blade $j$. This formula is valid for small difference between $\beta_{j}$ values. Notice that $\beta_{r}$ refers to reference values and $\beta_{m}$ refers to measured values of pitch angles.

In this benchmark model, the hydraulic pitch is piston servo mechanism witch can be modeled by a second order transfer function:

$$
\frac{\beta(s)}{\beta_{r}(s)}=\frac{\omega_{n}^{2}}{s^{2}+2 \xi \omega_{n} s+\omega_{n}^{2}}
$$

The model of the drive train system is given by the state space representation as

$$
\dot{x}_{d t}(t)=A_{d t}\left(\eta_{d t}\right) x_{d t}(t)+B_{d t} u_{d t}(t)
$$

where $x_{d t}=\left[\begin{array}{lll}\omega_{r} & \omega_{g} & \theta_{\Delta}\end{array}\right]^{T}$, is the state vector and $u_{d t}=$ $\left[\begin{array}{ll}\tau_{r} & \tau_{g}\end{array}\right]^{T}$, is the input vector of the drive train sub-system. $\omega_{r}$, is the rotor speed, $\omega_{g}$, is the generator speed, $\theta_{\Delta}$, is the torsion angle of the drive train and $\tau_{g}$, is the torque of the converter. $A_{d t}$ is modulated by a parameter of the drive train efficiency, $\eta_{d t}$, see Odgaard et al. [2009].

The converter dynamics can be modeled by a first order transfer function, consult Odgaard et al. [2009]:

$$
\frac{\tau_{g}(s)}{\tau_{g, r}(s)}=\frac{\alpha_{g c}}{s+\alpha_{g c}}
$$


The power produced by the generator is given by

$$
P_{g}(t)=\eta_{g} \omega_{g}(t) \tau_{g}(t)
$$

\subsection{Simulation Results}

The model of the real wind turbine is linearized for a power setpoint, $P_{r}$, of $4.8 M \mathrm{~W}$ and a wind speed, $v_{w}$, of $13 \mathrm{~m} / \mathrm{s}$. A white noise is added to wind speed sequence in ordre to simulate a real wind variations. The parameters of the wind turbine used are:

$R=57.5 ; \rho=1.225 ; \xi=0.6 ; \omega_{n}=11.11, \alpha_{g c}=50, \eta_{g}=0.98$. The considered turbine is subject to physical constraints on pitch angles, $\beta_{j}$.

$$
\mathscr{C}=\left\{\beta_{j} \in\left[0^{\circ} ; 90^{\circ}\right], j=1, \ldots, 3\right\}
$$

The sub-system to be studied in this work is the drive train system where the parameter of the efficiency decreases in function of the severity of the fault. Two cases are given below, the fault-free and post-fault model of the drive train and the controller are given also.

Fault-free case: In this case, the efficiency parameter, $\eta_{d t}$, for nominal functioning of the drive train system is fixed to 0.98 . So that, the state space model is

$$
\begin{gathered}
A_{d t}(0.98)=\left(\begin{array}{ccc}
-1.4229 * 10^{-5} & 1.4842 * 10^{-7} & -49.0909 \\
0.0203 & -0.1171 & 7.0688 * 10^{4} \\
1 & -0.0105 & 0
\end{array}\right) \\
B_{d t}=\left(\begin{array}{cc}
1.8182 * 10^{-8} & 0 \\
0 & -0.0026 \\
0 & 0
\end{array}\right)
\end{gathered}
$$

Besides, and after setting $Q$ and $R$ of the LQ controller to,

$$
\begin{gathered}
Q=100 * \operatorname{diag}([1,1,1,1,1,1,1,1,1,1]), \\
R=4 * \operatorname{diag}([21,21,21,1]),
\end{gathered}
$$

the feedback and feed-forward gains are $K=\left[\begin{array}{ll}K_{1} & K_{2}\end{array}\right]$ and $K_{r}$, where

$$
\begin{gathered}
K_{1}=\left(\begin{array}{rrrrr}
0.2829 & 3.2733 & 0.2380 & 3.2685 & 0.2380 \\
0.2380 & 3.2685 & 0.2829 & 3.2733 & 0.2380 \\
0.2380 & 3.2685 & 0.2380 & 3.2685 & 0.2829 \\
0.0077 & -0.1553 & 0.0077 & -0.1553 & 0.0077
\end{array}\right) \\
K_{2}=\left(\begin{array}{rrrrr}
3.2685 & -45.0414 & -0.2418 & 319.8854 & 0.0004 \\
3.2685 & -45.0414 & -0.2418 & 319.8854 & 0.0004 \\
3.2733 & -45.0414 & -0.2418 & 319.8854 & 0.0004 \\
-0.1553 & 15.7775 & -0.1725 & -258.3093 & 0.2498
\end{array}\right) \\
K_{r}=1.0 * 10^{-4}\left(\begin{array}{r}
-0.2183 \\
-0.2183 \\
-0.2183 \\
0.0453
\end{array}\right)
\end{gathered}
$$

Post-fault case: In this case, an actuator fault on the drive train system is considered at time $30 \mathrm{~s}$. This fault is reflected by the decrease of the efficiency parameter, $\eta_{d t}$, to 0.0828 value. The FDD stage in Fig. 3 is supposed perfect, so the faulty model of the drive train system is

$$
A_{d t}(0.0828)=\left(\begin{array}{crc}
-1.4229 * 10^{-5} & 1.4842 * 10^{-7} & -49.0909 \\
0.0017 & -0.1169 & 6.0340 * 10^{3} \\
1 & -0.0105 & 0
\end{array}\right)
$$

After the first reconfiguration action, the new controller gains are $K_{f}=\left[\begin{array}{ll}K_{f 1} & K_{f 2}\end{array}\right]$ and $K_{r}^{f}$, where

$$
\begin{aligned}
& K_{f 1}=\left(\begin{array}{rrrrr}
0.2606 & 2.9599 & 0.2157 & 2.9551 & 0.2157 \\
0.2157 & 2.9551 & 0.2606 & 2.9599 & 0.2157 \\
0.2157 & 2.9551 & 0.2157 & 2.9551 & 0.2606 \\
0.0089 & -0.0449 & 0.0089 & -0.0449 & 0.0089
\end{array}\right) \\
& K_{f 2}=\left(\begin{array}{rrrr}
3.2685-45.0414-0.2418 & 319.8854 & 0.0004 \\
3.2685-45.0414-0.2418 & 319.8854 & 0.0004 \\
3.2733-45.0414-0.2418 & 319.8854 & 0.0004 \\
-0.1553 & 15.7775-0.1725 & -258.3093 & 0.2498
\end{array}\right) \\
& K_{r}^{f}=1.0 * 10^{-4}\left(\begin{array}{r}
-0.2169 \\
-0.2169 \\
-0.2169 \\
0.0205
\end{array}\right)
\end{aligned}
$$

Notice that, for reason of paper width, $K$ and $K_{f}$ are divided into two matrices $K_{1}, K_{2}$ and $K_{f 1}, K_{f 2}$ respectively.

The simulation results of the wind turbine output are shown in Fig. 5. Before the fault occurrence, the plant can track the nominal set-point of power, $4.810^{6} \mathrm{~W}$. After the fault occurrence at $30 \mathrm{~s}$, the efficiency parameter of the drive train system decreases enormously and the plant performance is degraded respectively which requires to reconfigure the setpoint. The set-point reconfiguration is parameterized by the fault severity and by the plant constraints which become more probable to be violated (see Fig. 6).

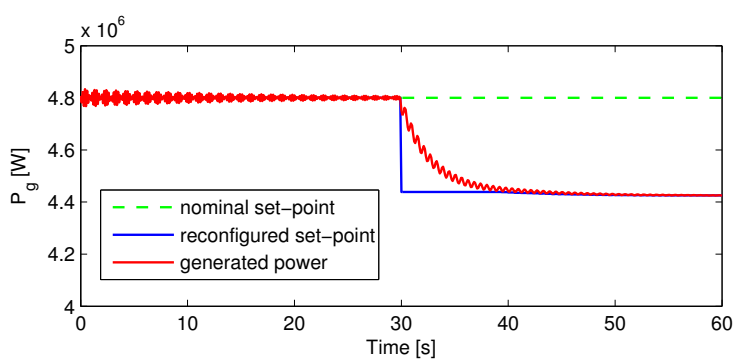

Fig. 5. The generated electrical power by the wind turbine.
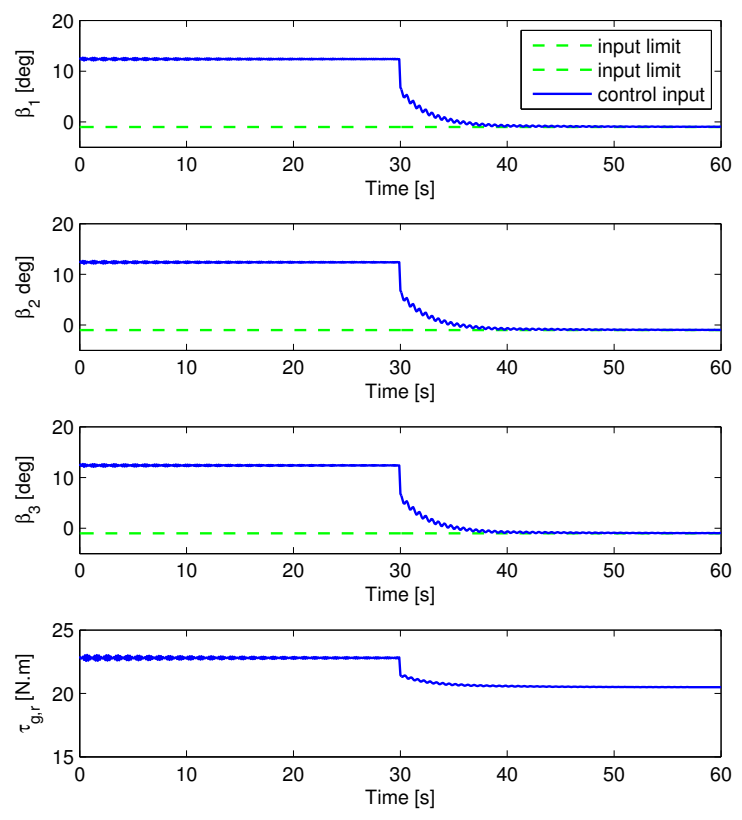

Fig. 6. The 3 pitch angles and the generator torque controls. 
In Fig. 6, and before the fault occurrence, the control inputs are inside the control limits required by the constraints set $\mathscr{C}$ in (37). However, and after the fault occurrence, the pitch angles decreases in order to compensate the fault effect to their minimal value without compensating the fault. The second action of reconfiguration is needed now in order keep the plant operating with degraded performance: the set-point reconfiguration. This action is ensured by the ROG unit like as mentioned in Fig. 3.

\subsection{Performance evaluation}

To evaluate the performance of the closed-loop system, one could use the following performance index

$$
\pi(t)=1-\frac{\|y(t)-r(t)\|_{2}}{\|r(t)\|_{2}}
$$

where $r$ is the reference or the nominal set-point and $y$ is the regulated output. $\|x\|_{2}$ is the norm 2 of $x$.

Fig. 7 shows the evolution of the performance index, $\pi$, of the plant before and after the fault occurrence. This index can help the supervisor to monitor the plant by fixing a threshold for admissible performance degradation. The selection of the admissible threshold for acceptable performance degradations depends on safety and quality requirements. In this case of study, the maximal reference tracking error is 0.0782 (about $8 \%$ ) which is acceptable if the selected threshold of $\pi$ is $90 \%$.

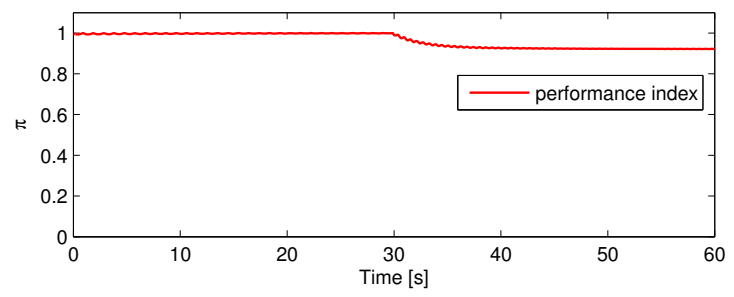

Fig. 7. The performance index in-time evolution.

\section{CONCLUSION}

This paper presents a new approach to fault accommodation with performance degradation based on Reference-Offset Governor. The degraded ROG based FTC modify the set-points and add an offset to the control inputs after fault occurrence in order to accommodate the fault with acceptable degraded performance. The degradation of performance is supervised by the operator through a performance index. The simulation results proof that this approach improves the system performance degradation and ensure a safe and stable plant operation.

\section{REFERENCES}

D. Angeli, A. Casavola and E. Mosca. On feasible setmembership state estimators in constrained command governor control. Automatica, vol. 37:pp. 151-156, 2001.

C. Aubrun, P. De Cuypere and D. Sauter. Design of a supervised control system for sludge dewatering process. Control Eng. Practice, vol. 11:pp. 27-37, 2003.

A. Bemporad, A. Casavola and E. Mosca. Nonlinear Control of Constrained Linear Systems via Predictive Reference Management. IEEE Transactions on Automatic Control, vol. 42: pp. 340-349, 1997.
A. Casavola, E. Mosca and D. Angeli. Robust Command Governors for Constrained Linear System. IEEE Transactions on Automatic Control, vol. 45:pp. 2071-2077, 2000.

A. Casavola, M. Papini and G. Franz. Supervision of Networked Dynamical Systems Under Coordination constraints. IEEE Transactions on Automatic Control, vol. 51, No. 3:pp. pp. 421-437, 2006.

A. Casavola, G. Franz and M. Sorbara. "Reference-Offset Governor approach for the supervision of constrained networked dynamical systems". in European Control Conference, Kios, Greece, pp. 7-14, 2007.

E. Gilbert, I. Kalmanovsky and K. Tan. Discrete-time reference governors and the nonlinear control of systems with state and control constraints. Int. J. on Robust and Nonlinear Control, vol. 5:pp. 487-504, 1995.

E. Gilbert and K. Tan. Linear Systems with State and Control Constraints: The theory and Application of Maximal Output Admissible Sets. IEEE Transactions on Automatic Control, vol. 36:pp. 1008-1020, 1991.

O. Harkegard and S. Glad. Resolving actuator redundancy optimal control vs. control allocation. Automatica, vol. 41: pp. 137-144, 2005.

J. Jiang and Y. Zhang. "Graceful performance degradation in active fault tolerant control systems". In Proc. of the 15th IFAC World Congress b'02, Barcelona, Spain, 2002.

J. Jiang and Y. Zhang Accepting Performance Degradation in Fault-Tolerant Control System Design. IEEE Transactions on Control Systems Technology, vol. 14:pp. 284-292, 2006.

P. Kapasouris, M. Athans and G. Stein "Design of feedback control systems for stable plants with saturating actuators". in Proc. 27th IEEE Conference on Decision and Control, Austin, Texas, USA, pp. 469-479, 1988.

I. Kolmanovsky and J. Sun. Parameter governors for discretetime nonlinear systems with pointwise-in-time state and control constraints. Automatica, vol. 42:pp. 841-848, 2006.

K. Johnson, M. Pao, L.Y. Balas and L. Fingeresh. Control of variable-speed wind turbines - standard and adaptative techniques for maximizing energy capture. IEEE Control Systems Magazine, vol. 26:pp. 70-81, 2006.

M. V. Kothare, and M. Morari. Multiplier theory for stability analysis of anti-windup control systems. Automatica, vol. 35:pp. 917-928, 1999.

Q. Mayne, B. Rawlings, V. Rao and M. Scokaert. Constrained model predictive control: stability and optimality. Automatica, vol. 36, No. 6:pp. 789-814, 2000.

P.F. Odgaard, J. Stoustrup and M. Kinnaert. 'Fault Tolerant Control of Wind Turbines - a benchmark model". in Proc. of the 7th IFAC Symposium on Fault Detection, Supervision and Safety of Technical Processes, Barcelona, Spain, pp. 155-160, 2009.

M. Staroswiecki. "Actuator faults and the linear quadratic control problem". in Proc. of the 42th IEEE Conference on Decision and Control, Maui, Hawaii, USA, pp. 959-965, 2003.

Y. Zhang and J. Jiang. Fault Tolerant Control System Design with Explicit Consideration of Performance Degradation. IEEE Transactions on Aerospace and Electronic Systems, vol. 39:pp. 838-844, 2003.

F. Wu and B. Lu Anti-windup control design for exponentially unstable LTI systems with actuator saturation. Systems \& Control Letters, vol. 52:pp. 305-322, 2004. 